\title{
Performance of field-emitting resonating carbon nanotubes as radio-frequency demodulators
}

\author{
P. Vincent,,${ }^{1, *}$ P. Poncharal, ${ }^{1}$ T. Barois,${ }^{1}$ S. Perisanu, ${ }^{1}$ V. Gouttenoire, ${ }^{1}$ H. Frachon, ${ }^{1}$ A. Lazarus,${ }^{2}$ E. de Langre, ${ }^{2}$ E. Minoux, ${ }^{3}$ \\ M. Charles, ${ }^{3}$ A. Ziaei, ${ }^{3}$ D. Guillot, ${ }^{1}$ M. Choueib,${ }^{1}$ A. Ayari, ${ }^{1}$ and S. T. Purcell ${ }^{1}$ \\ ${ }^{1}$ Laboratoire de Physique de la Matière Condensée et Nanostructures, Université Lyon 1, CNRS, UMR 5586, Domaine Scientifique de la \\ Doua, F-69622 Villeurbanne Cedex, France \\ ${ }^{2}$ Laboratoire d' Hydrodynamique, Ecole Polytechnique, F-91128 Palaiseau, France \\ ${ }^{3}$ Thales Research \& Technology France. 1, avenue Augustin Fresnel, Campus de Polytechnique, F-91767 Palaiseau cedex, France
}

(Received 9 November 2010; published 26 April 2011)

\begin{abstract}
We report on a systematic study of the use of resonating nanotubes in a field emission (FE) configuration to demodulate radio frequency signals. We particularly concentrate on how the demodulation depends on the variation of the field amplification factor during resonance. Analytical formulas describing the demodulation are derived as functions of the system parameters. Experiments using AM and FM demodulations in a transmission electron microscope are also presented with a determination of all the pertinent experimental parameters. Finally we discuss the use of CNTs undergoing FE as nanoantennae and the different geometries that could be used for optimization and implementation.
\end{abstract}

DOI: 10.1103/PhysRevB.83.155446

PACS number(s): 85.85.+j, 61.48.De, 79.70.+q, 84.30.Qi

\section{INTRODUCTION}

An aspect of using carbon nanotubes and nanowires (CNTs/NWs $\equiv \mathrm{NNs}$ ) as electron field emission (FE) sources is that their mechanical resonances can be excited simultaneously to field emission by applying an additional low amplitude ac voltage at the resonance frequency. We previously reported the main aspects of mechanical excitation of NNs during $\mathrm{FE}^{1,2}$ : (1) the mechanical resonances are observed directly in the FE microscopy (FEM) patterns by a widening in the vibration direction; (2) the resonance frequencies can be tuned by a large factor by the dc applied voltage because of the electrostatic stretching of these nanocantilevers; and (3) the FE current has an ac component that is enhanced at the mechanical resonance and a modification of the average current due to the strong nonlinearity of the field emission. This is due to the dependence of the field amplification factor $\beta(y)$ that controls the field emission current through the Fowler-Nordheim (FN) equation on the relative transverse position $y$ of the $\mathrm{NN}$ apex.

Several original applications based on the combination of mechanics and FE for NNs have recently been demonstrated including position sensors, ${ }^{3}$ atomic mass sensing, ${ }^{4}$ self-oscillating ac nanogenerators, ${ }^{5}$ and the $\mathrm{FE}$ electron shuttle. ${ }^{6}$ More particularly for this article, Jensen and co-workers have nicely demonstrated a fully integrated radio receiver, termed the "nanoradio", 7 consisting of a single CNT in FE configuration that can simultaneously play the role of four essential components of a radio receiver: the antenna, the tuner, the amplifier, and the demodulator. They performed demodulation of both frequency modulated (FM) and amplitude modulated (AM) signals and their nanoradio was used successfully for music reception. Their experiments were performed in a transmission electron microscope (TEM) that allowed characterization of the nanotube mechanical motion during radio reception. This extremely compact radio has been attracting a great deal of attention and opens potential applications in smart $\mathrm{dust}^{8}$ components, enhanced radio frequency identification (RFID) tags, or simply smaller and cheaper wireless devices such as cellular phones.

These recent observations of the decoding of audio signals are encouraging, but there is unfortunately neither a clear understanding of the underlying mechanism nor the overall geometry to enable reliable, nanoradio devices. First there is a systematic lack of the experimental parameters such as emission voltage, total current, demodulated current, etc., that could permit comparisons with other experiments or to estimate the overall performance. A more fundamental point is that in their brief analysis of the demodulation, the main effect is considered to be due to the linear dependence of the field amplification factor on the relative position of the CNT apex. We show here that this misses the main demodulating term which actually depends quadratically on the position. The correct analysis of the demodulation is essential for optimizing the device geometry. Furthermore, we show that the coupling between the emitting CNT and the incoming electromagnetic wave is much weaker than originally suggested and therefore it is very difficult to use the CNT itself as an antenna in this configuration. Rather, the CNT has to be excited capacitively by an electrical wave provided for example by an external antenna.

The article describes further theoretical and experimental results on the FE demodulation process by individual CNTs in different configurations. The first analytical part focuses on the dependence of the field amplification factor on the CNT apex position since it is the key parameter for the demodulation process. This is accompanied by an analysis of the different contributions to the demodulated current in AM or FM coding. In the experimental part we repeat the experiments of Jensen et al. by carrying out both AM and FM demodulation experiments with a CNT under FE in a TEM. The different parameters of the nanoradio are quantified and they are used for analytical calculation of the measured signals. Finally we discuss the possibility of using CNTs as antennae, the general performances of this nanoradio as well as how other geometry could be used for improvement. 


\section{THEORY}

In the CNT FE nanoradio the demodulation is provided by the coupling between the rf voltage signal, the mechanical response of the CNT, and the field emission current. The rf signal creates oscillating forces on the CNT which vibrates when the driving and resonance frequencies match. These oscillations vary the field $F$ at the CNT apex given by $F=\beta(y) V_{A}$ where $\beta(y)$ is the field amplification factor which quantifies the tip effect and $V_{A}$ is the applied voltage. For an oscillating CNT to first order, the variations in $\beta(y)$ depend on the transverse displacement of the apex (defined as $y$ ). The oscillations in $F$ cause oscillations in the FE current which varies as $I_{\mathrm{FE}}(t) \alpha \exp [-\mathrm{const} / F(t)]$. The nonlinear response creates harmonic signals, including the low-frequency modulation signal, whose relative importance depends on the variation of $\beta(y)$ around the $\mathrm{CNT}$ rest position. In the following we will analyze the frequency response in detail and discuss the different contributions that can be exploited for improving the demodulation.

The mechanical excitation of CNTs by pure sinusoidal high-frequency signals has been investigated in TEM, ${ }^{9}$ scanning electron microscopy (SEM), ${ }^{10}$ and FE environments ${ }^{1,11,12}$ to obtain the angular resonance frequency $\omega_{0}$ (denoted as frequency in the following), the Young's modulus $Y$, and the quality factors $Q$ of different CNTs and NWs. For a linear mechanical response one has the well-known Lorentzian oscillation amplitude given by (also shown in blue in Fig. 1)

$$
y(\omega)=\frac{T}{\sqrt{\left(\omega_{0}^{2}-\omega^{2}\right)^{2}+\left(\frac{\omega_{0} \omega}{Q}\right)^{2}}},
$$

where $T$ is the transverse excitation force amplitude per effective unit mass $\left(\mathrm{m} \mathrm{s}^{-2}\right)$ due to the imbalance of electric fields over the whole CNT surface. It is zero for a perfectly

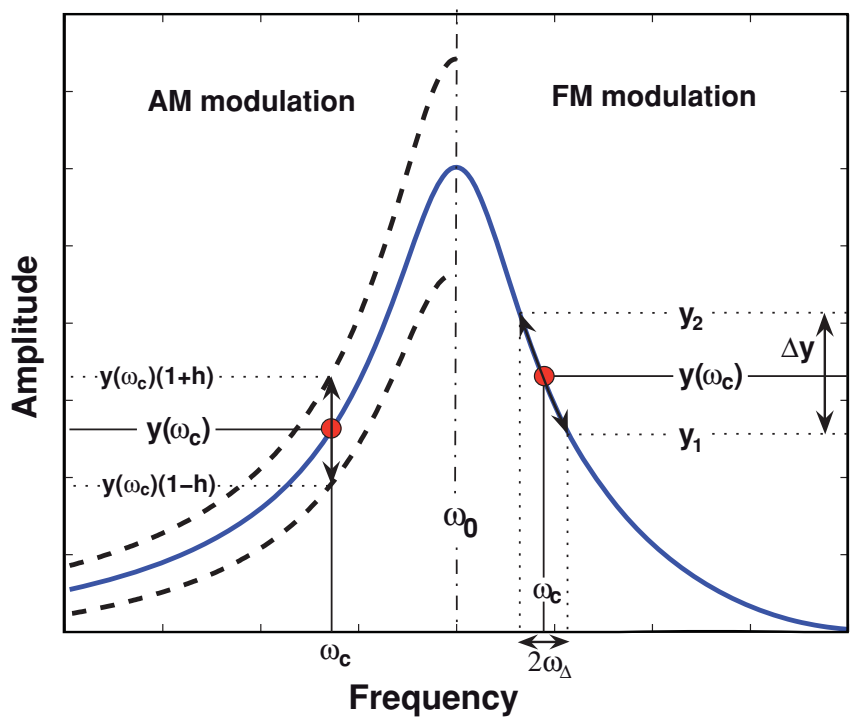

FIG. 1. (Color online) Schematic showing the demodulation process across the mechanical resonance of a CNT. The solid line (blue) traces the linear mechanical amplitude vs the excitation frequency. Left (right) schematizes the AM (FM) demodulation process. The AM and FM signals result in low-frequency variations of the oscillations that cause in turn variations of the $\beta(y)$. symmetric configuration. This symmetry is broken if the CNT, the support tip, or the counter electrode have nonzero tilt angles for the rest position or if the CNT is structurally asymmetric.

A pure cosine low frequency modulating signal is used in the analysis (a pure note). For AM modulation the rf signal is then given by $V_{\mathrm{AM}}(t)=A\left[1+h \cos \left(\omega_{L} t\right)\right] \cos \left(\omega_{c} t\right)$, where $\omega_{L}$ is the frequency of the modulating signal, $\omega_{c}$ is the carrier frequency, and $h$ is called the modulation index $(h<1)$ which varies the strength of the modulation. In FM the modulation is introduced via the phase $\phi(t)$ of the rf signal $V_{\mathrm{FM}}(t)=$ $A \cos [\phi(t)]$ by the relation $d \phi / d t=\omega_{i}=\omega_{c}+\omega_{\Delta} \cos \left(\omega_{L} t\right)$, where $\omega_{i}$ is the instantaneous frequency, $\omega_{c}$ is again the carrier frequency, and $\omega_{\Delta}$ is the frequency deviation that corresponds to the maximum deviation of the instantaneous frequency with respect to the carrier frequency. Integrating $d \phi / d t$ one obtains for the FM signal $V_{\mathrm{FM}}(t)=A \cos \left[\omega_{c} t+\omega_{\Delta} / \omega_{L} \sin \left(\omega_{L} t\right)\right]$.

It is well known in electronics that a nonlinear device, such as a diode or an envelope detector, is sufficient to recover the low-frequency part of an AM signal. Thus in principle the strongly nonlinear FE characteristic is sufficient for demodulation. For FM demodulation a specific component must be used whose output varies linearly with the instantaneous frequency of the incoming signal. The simplest form of FM detector is a slope detector such as a tank circuit which first converts the FM to AM. This AM signal is then demodulated as before. The main difference in a FE nanoradio is that a supplementary mechanical stage, the mechanical resonator, is inserted between the electrical excitation and the final demodulated current. This leads to original terms in the mathematical description of both AM and FM demodulation that strongly increase the resulting demodulated currents.

The effect of the rf modulated signal on the oscillation when the carrier frequency matches the resonance is illustrated in Fig. 1 for both AM (left of the figure) and FM (right). For AM, the carrier frequency being constant, the oscillation amplitude varies at $\omega_{L}$. For FM the excitation frequency slowly oscillates around the carrier frequency and the amplitude of oscillation follows $y(\omega)$, resulting in an amplitude modulated oscillation. This is similar to an electronic slope detector though the modulation is now mechanical and not electrical.

We now apply standard small signal analysis for $y(\omega)$. For a given carrier frequency and modulation index the time-dependent amplitude of oscillation for an AM signal is given by

$$
y(t)=y\left(\omega_{c}\right)\left[1+h \cos \left(\omega_{L} t\right)\right] \cos \left[\omega_{c} t+\phi\left(\omega_{c}\right)\right],
$$

where $\phi\left(\omega_{c}\right)$ is the phase shift between the mechanical oscillation and the excitation signal.

For FM let us consider small frequency deviations as shown in Fig. 1 somewhat off the resonance maximum. It is more convenient to rewrite the excitation signal as $V_{\mathrm{FM}}(t)=A \cos \left(\omega_{i} t\right)$ and to consider that the CNT follows the instantaneous frequency $\omega_{i}$. This approximation is correct here since the transients disappear very rapidly compared to the low-frequency modulation. We can then write the evolution of amplitude as (see Fig. 1)

$$
y(t)=\left[y\left(\omega_{c}\right)+y^{\prime}\left(\omega_{c}\right) \omega_{\Delta} \cos \left(\omega_{L} t\right)\right] \cos \left[\omega_{i} t+\phi\left(\omega_{i}\right)\right],
$$


where $y^{\prime}\left(\omega_{c}\right)$ is the first derivative of the amplitude with respect to frequency. Thus the FM translates into AM with $h$ replaced by $y^{\prime}\left(\omega_{c}\right) \omega_{\Delta} / y\left(\omega_{c}\right)$.

The next step is to consider how the mechanical oscillations modify the field amplification factor $\beta(y)$. This turns out to be somewhat difficult to measure or to simulate. First $\beta(y)$ depends on all the dimensions of the cathode/anode system, particularly the near apex geometry, and second it depends strongly on the precise form of the CNT apex which can be quite complicated, often terminating with nanoprotrusions. As well, $\beta(y)$ for the nontreated emitters and modest vacuum generally used in these experiments evolves continually in time due to mass transport to and from the apex region. Experimentally it would be necessary to measure the FE current variation during vibration at high frequency which is very difficult and not within the scope of this article. In summary, at this time we use a simple hemispherical end for the CNTs.

Though detailed simulations are presented below we first consider the Taylor expansion of $\beta(y)$ around the CNT apex rest position:

$$
\beta(y)=\beta_{0}+\beta_{1} y+\beta_{2} y^{2}+O\left(y^{2}\right),
$$

$\beta_{1}$ and $2 \beta_{2}$ are the first and second spatial derivatives. They depend strongly on the local environment of the CNT and give different contributions to the demodulated current. In particular, it is straightforward that in a symmetric configuration all the odd terms in the development vanish and thus the first term to consider is the second-order term. This term is missing in the analysis of Jensen et al. The second-order term is in fact particularly interesting since its nonlinear dependence has a strong demodulation effect.

Substituting Eqs. (2) or (3) into (4) one sees that the $\beta_{1} y$ term gives rise to a high-frequency modulated variation although the $\beta_{2} y^{2}$ term contains a demodulated component. In the case of Eq. (3) one gets

$$
\begin{aligned}
\beta_{2} y^{2} & =\beta_{2}\left[y\left(\omega_{c}\right)+y^{\prime}\left(\omega_{c}\right) \omega_{\Delta} \cos \left(\omega_{L} t\right)\right]^{2} \cos ^{2}\left[\omega_{i} t+\phi\left(\omega_{i}\right)\right] \\
& =\mathrm{cst}+\beta_{2} y\left(\omega_{c}\right) y^{\prime}\left(\omega_{c}\right) \omega_{\Delta} \cos \left(\omega_{L} t\right) \\
& +\frac{\beta_{2}}{4} y^{\prime 2}\left(\omega_{c}\right) \omega_{\Delta}^{2} \cos \left(2 \omega_{L} t\right)+\text { HF terms }
\end{aligned}
$$

That corresponds to a constant term plus a low frequency signal at $\omega_{L}$ (the demodulated signal), $\beta_{2} y\left(\omega_{c}\right) y^{\prime}\left(\omega_{c}\right) \omega_{\Delta} \cos \left(\omega_{L} t\right)$, a low frequency signal at $2 \omega_{L}$ that is the harmonic distortion altering the quality of the signal and high-frequency components around $2 \omega_{i}$. The main point here is the appearance of a demodulated component in the variation of $\beta$ that in turn will lead to a direct demodulation current.

The final step is to re-inject these variations into the FowlerNordheim equation. Here we take a simple but sufficient approximation of the current under the form

$$
I(V, y)=A \beta(y)^{2} V^{2} \exp \left[\frac{-B}{\beta(y) V}\right] .
$$

Defining $d V$ as the $V_{\mathrm{AM}}$ or $V_{\mathrm{FM}}$ signal and $d \beta$ as the field amplification factor variation given by $d \beta=\beta(y)-\beta_{0}$ we can develop $I(t)$ by a Taylor expansion that gives

$$
\begin{aligned}
I(t)= & I_{0}+\left.\frac{\partial I}{\partial V}\right|_{V_{\mathrm{dc}}} d V+\left.\frac{\partial I}{\partial \beta}\right|_{\beta_{0}} d \beta+\left.\frac{\partial^{2} I}{\partial V^{2}}\right|_{V_{\mathrm{dc}}} \frac{d V^{2}}{2} \\
& +\left.\frac{\partial^{2} I}{\partial^{2} \beta}\right|_{\beta_{0}} \frac{d \beta^{2}}{2}+\left.\frac{\partial^{2} I}{\partial \beta \partial V}\right|_{V_{\mathrm{dc}}, \beta_{0}} d \beta d V,
\end{aligned}
$$

where $I_{0}=I\left(V_{\mathrm{dc}}, \beta_{0}\right)$ and the different partial derivatives can be expressed in terms of FE parameters

$$
\begin{gathered}
\left.\frac{\partial I}{\partial V}\right|_{V_{\mathrm{dc}}}=I_{0}\left(\frac{2}{V_{\mathrm{dc}}}+\frac{B}{\beta_{0} V_{\mathrm{dc}}^{2}}\right), \\
\left.\frac{\partial I}{\partial \beta}\right|_{\beta_{0}}=I_{0}\left(\frac{2}{\beta_{0}}+\frac{B}{\beta_{0}^{2} V_{\mathrm{dc}}}\right), \\
\left.\frac{\partial^{2} I}{\partial V^{2}}\right|_{V_{\mathrm{dc}}}=I_{0}\left(\frac{2}{V_{\mathrm{dc}}^{2}}+\frac{2 B}{\beta_{0} V_{\mathrm{dc}}^{3}}+\frac{B^{2}}{\beta_{0}^{2} V_{\mathrm{dc}}^{4}}\right), \\
\left.\frac{\partial^{2} I}{\partial \beta^{2}}\right|_{\beta_{0}}=I_{0}\left(\frac{2}{\beta_{0}^{2}}+\frac{2 B}{\beta_{0}^{3} V_{\mathrm{dc}}}+\frac{B^{2}}{\beta_{0}^{4} V_{\mathrm{dc}}^{2}}\right), \\
\left.\frac{\partial^{2} I}{\partial \beta \partial V}\right|_{V_{\mathrm{dc}}, \beta_{0}}=I_{0}\left(\frac{4}{\beta_{0} V_{\mathrm{dc}}}+\frac{3 B}{\beta_{0}^{2} V_{\mathrm{dc}}^{2}}+\frac{B^{2}}{\beta_{0}^{3} V_{\mathrm{dc}}^{3}}\right) .
\end{gathered}
$$

The different terms that will contribute to demodulation are contained in Eq. (7). The first interesting term for AM is the $d \beta$ one since its $\beta_{2} y^{2}$ factor contains a demodulated component. The $d V^{2}$ also contributes nonlinearly, the $d \beta^{2}$ demodulates partly due to the $\beta_{1} y$ dependence, and finally the $d \beta d V$ also contributes. These additional mechanical resonance terms greatly improve the demodulation performances.

The $d \beta$ term is still present for FM for the same reason as before but the $d V^{2}$ term does not contribute this time. The $d \beta^{2}$ and $d \beta d V$ terms also demodulate.

Equations (2), (3), and (4) can be used to directly estimate for each term of Eq. (7) the amplitude of the demodulated current. After removing the $\cos \left(\omega_{L} t\right)$ factors this gives the following AM components:

$$
\begin{gathered}
d \beta: I_{0}\left(\frac{2}{\beta_{0}}+\frac{B}{\beta_{0}^{2} V_{\mathrm{dc}}}\right) h \beta_{2} y\left(w_{c}\right)^{2}, \\
d \beta^{2}: I_{0}\left(\frac{2}{\beta_{0}^{2}}+\frac{2 B}{\beta_{0}^{3} V_{\mathrm{dc}}}+\frac{B^{2}}{\beta_{0}^{4} V_{\mathrm{dc}}^{2}}\right)\left\{h \left[\beta_{1}^{2} y\left(\omega_{c}\right)^{2}\right.\right. \\
\left.\left.+3 \beta_{2}^{2} y\left(\omega_{c}\right)^{4}\right]+h^{3} \frac{9}{8} \beta_{2}^{2} y\left(\omega_{c}\right)^{4}\right\} \\
d V^{2}: I_{0}\left(\frac{2}{V_{\mathrm{dc}}^{2}}+\frac{2 B}{\beta_{0} V_{\mathrm{dc}}^{3}}+\frac{B^{2}}{\beta_{0}^{2} V_{\mathrm{dc}}^{4}}\right) \frac{V_{\mathrm{ac}}^{2}}{2} \\
d \beta d V: I_{0}\left(\frac{4}{\beta_{0} V_{\mathrm{dc}}}+\frac{3 B}{\beta_{0}^{2} V_{\mathrm{dc}}^{2}}+\frac{B^{2}}{\beta_{0}^{3} V_{\mathrm{dc}}^{3}}\right) \beta_{1} \\
y_{R}\left(\omega_{c}\right) h \frac{V_{\mathrm{ac}}}{2}
\end{gathered}
$$

$y_{R}\left(\omega_{c}\right)$ is the in-phase response function. This function appears here because in the $d \beta d V$ term the response function is multiplied by the excitation signal which involves the real part of the transfer function.

For FM demodulation the same kind of analysis gives

$$
d \beta: I_{0}\left(\frac{2}{\beta_{0}}+\frac{B}{\beta_{0}^{2} V_{d c}}\right) \beta_{2} y\left(w_{c}\right) y^{\prime}\left(w_{c}\right) \omega_{\Delta},
$$




$$
\begin{gathered}
d \beta^{2}: I_{0}\left(\frac{2}{\beta_{0}^{2}}+\frac{2 B}{\beta_{0}^{3} V_{d c}}+\frac{B^{2}}{\beta_{0}^{4} V_{d c}^{2}}\right) y\left(\omega_{c}\right) \omega_{\Delta}\left[\beta_{1}^{2} y^{\prime}\left(\omega_{c}\right)\right. \\
\left.+\frac{3}{2} \beta_{2}^{2} y\left(\omega_{c}\right)^{2} y^{\prime}\left(\omega_{c}\right)+\frac{9}{8} \beta_{2}^{2} y^{\prime}\left(\omega_{c}\right)^{3} \omega_{\Delta}^{2}\right] \\
d \beta d V: I_{0}\left(\frac{4}{\beta_{0} V_{d c}}+\frac{3 B}{\beta_{0}^{2} V_{d c}^{2}}+\frac{B^{2}}{\beta_{0}^{3} V_{d c}^{3}}\right) \beta_{1} \\
y_{R}^{\prime}\left(\omega_{c}\right) \omega_{\Delta} \frac{V_{a c}}{2}
\end{gathered}
$$

$y_{R}^{\prime}\left(\omega_{c}\right)$ is the first derivative of the in-phase response function.

These formulas give the different contributions of the demodulated current as a function of the field emission and mechanical properties of the CNT, the CNT environment, and the rf parameters. Since many parameters are needed to describe the system, large order of magnitude errors in the estimation of the demodulated current can easily occur and consequently an important effort must be made to measure and/or estimate them well. In situ experiments of the nanoradio were carried out in a TEM on different CNTs to obtain experimental values and good approximations of all the terms.

\section{EXPERIMENT}

We used arc-discharge multiwall carbon nanotubes glued at the apexes of tungsten tips. These CNT tips were then mounted in a homemade TEM sample holder to simultaneously observe the motion of the CNTs and the demodulation process [see Fig. 2(a)]. The CNT tip was connected to a heating loop that could be floated to high voltage $(2000 \mathrm{~V})$ and positioned near a gold ball that is both the extraction anode and the emission current collector. This anode was mounted on a micrometric movement that could be used to change the nanotube-anode distance. An additional excitation electrode with $50 \Omega$ impedance was positioned near the nanotube. rf signals were sent to either the tip, the gold ball or the excitation electrode depending on the experimental conditions. This TEM holder was made to be inserted into a TOPCON EM002b microscope with large gap pole pieces which limit the resolution of the images to about $0.4 \mathrm{~nm}$ at $120 \mathrm{kV}$.

Preliminary observations were then realized to characterize the CNT dimensions [Fig. 2(b)]. The FE performances of the CNT were first determined. The emission voltage is extremely sensitive to the nanotube-anode distance and nanotube dimensions. To partially normalize the experiments on different nanotubes the anode position was adjusted to obtain a current of $10 \mu \mathrm{A}$ for nearly $300 \mathrm{~V}$ [see Fig. 2(d), the electronic caustic at the apex of the CNT is due to the high electric field]. Once this distance was established, the system was kept constant. The $I(V)$ characteristics shown in Fig. 3 that follow the FN equation were then obtained and they were used for our nanoradio. Using a work function $\phi$ of $5 \mathrm{eV}$ and taking $B \simeq-0.683 \phi^{3 / 2} v(y) \simeq 6.11 \times 10^{10} \mathrm{~V} \mathrm{~m}^{-1}$ the experimental curves could be fitted to determine the amplification factor $\beta_{0}$ and the constant $A$ in Eq. (6). This gave $\beta_{0}=2.905 \times 10^{7}$ $\mathrm{m}^{-1}$ and $A=1.212 \times 10^{-22} \mathrm{~A} \mathrm{~V}^{-2} \mathrm{~m}^{2}$. It should be noted that CNTs that were not well glued to their tungsten tips could be torn off during these preliminary FE experiments.

The resonance frequencies versus the applied voltage were determined next and the quality factor $Q$ was measured at

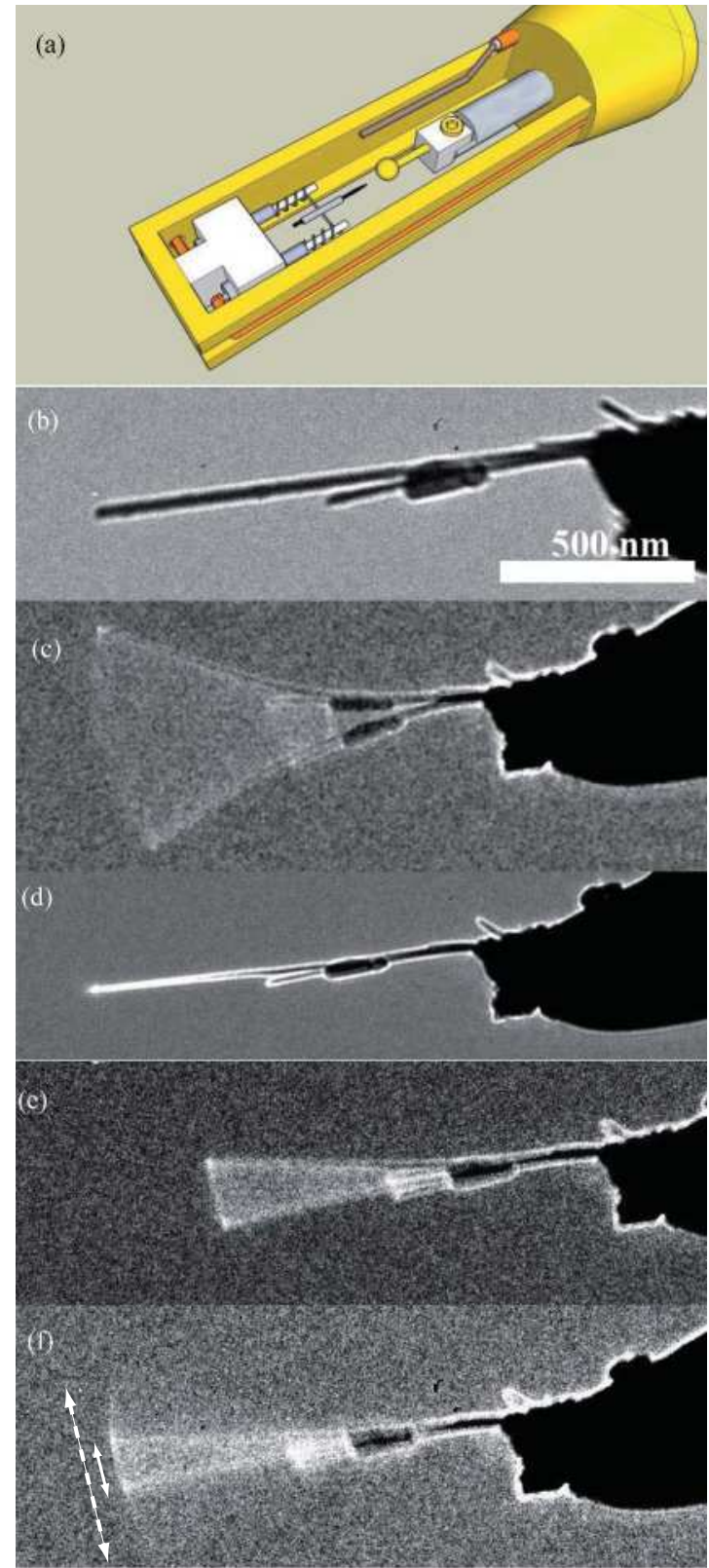

FIG. 2. (Color online) Experimental schema and TEM observations during the nanoradio experiments. (a) Schema of the homemade TEM holder where one distinguishes the tungsten tip positioned in front of the gold ball that was used as an anode and the additional excitation anode. (b) Observation of one mutiwalled CNT used for these experiments. The CNT has a length of $1.3 \mu \mathrm{m}$ and a diameter of nearly $30 \mathrm{~nm}$. (c) Excitation of the first-resonance frequency. Large vibration amplitudes up to $400 \mathrm{~nm}$ can be obtained. (d) Field emission characterization of the CNT (the bright spot at the apex is due to the strong electric field). The anode-CNT distance was modified to obtain currents in the microampere range for $300 \mathrm{~V}$. (e) CNT vibrations during $\mathrm{FE}$ to determine the dependence of resonance frequency on dc voltage. (f) Nanoradio experiments. Here the CNT is excited by an rf signal with a FM coding of a pure note. Two limit envelopes of the oscillations are observed corresponding to the two extreme oscillation amplitudes (represented by $y_{1}$ and $y_{2}$ in Fig. 1).

low excitation (see Fig. 4). This gave the linear response of the cantilever that could be fit with a Lorentzian shape 

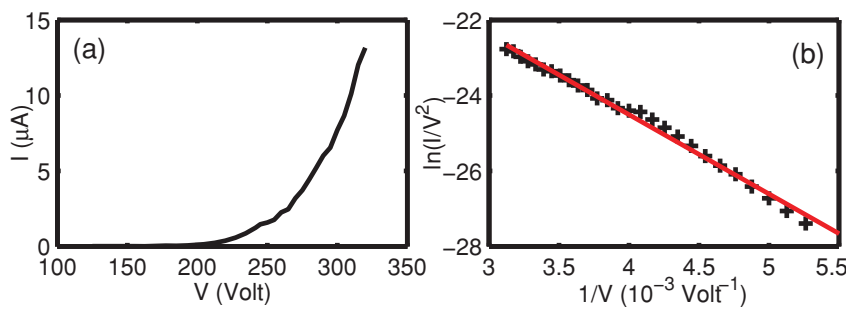

FIG. 3. (Color online) Field emission characterization of the CNT presented in Fig. 2. The anode-nanotube distance has been optimized to obtain a current in the microampere range for a few hundreds of volts. (a) Linear representation. (b) FN representation with the fitted characteristic that gives the field amplification factor $\beta_{0}$ and the constant $A$ in Eq. (6).

of the form stated in Eq. (1). The CNT presented in Fig. 2 had a resonant frequency of $f_{0}=\omega_{0} / 2 \pi=36.83 \mathrm{MHz}$ at zero applied voltage, a quality factor of around 800 That is rather large despite the small extra nanotube stuck on the main nanotube, and $T=3.95 \times 10^{6} \mathrm{~m} \mathrm{~s}^{-2}$. This gives both the amplitude frequency response $y\left(\omega_{c}\right)$ and its first derivative $y^{\prime}\left(\omega_{c}\right)$. Very high amplitudes, up to $400 \mathrm{~nm}$, could be obtained as seen in Fig. 2(c) which was interesting for getting good electromechanical demodulation performance.

After the CNT was fully characterized the nanoradio experiments were carried out. A Rohde and Schwarz SML 01 signal generator was used that permitted AM or FM modulation from external ports. We applied a dc voltage of $300 \mathrm{~V}$ giving an emission current in the microampere range and found a frequency electrostatically tuned to $82 \mathrm{MHz}$. The rf modulated signal was then sent to the nanotube for demodulation and the carrier frequency was swept to obtain the best demodulation rate. The emission current was collected at the anode and sent, via a dc block to remove the dc current, to a low-noise transimpedance amplifier (with a frequency cutoff at $100 \mathrm{kHz}$ ). The amplified signal was finally connected to a loud speaker for signal restitution (see Fig. 5).

Demodulation of an AM signal was rather easy to obtain for the first tests using $h=0.9$ and $V_{\mathrm{ac}}=0.2 \mathrm{~V}$. Away from

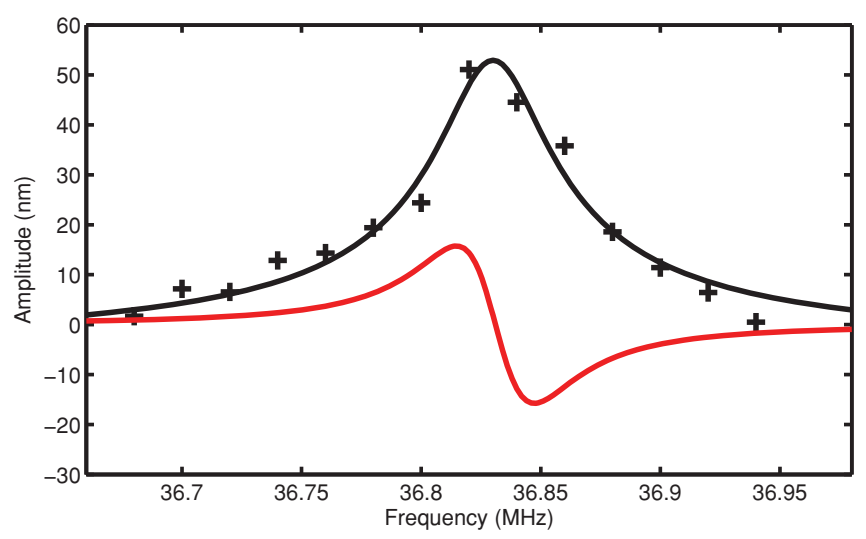

FIG. 4. (Color online) Experimental (cross points) and fitted (black curve) amplitude frequency response of the CNT presented in Fig. 2 at zero applied voltage. This gave a resonance frequency of $36.83 \mathrm{MHz}$ and a quality factor of 800 . This curve allows the calculation of the the first derivative $y^{\prime}\left(\omega_{c}\right)$ presented in the real part of the response function (red curve, not to scale).

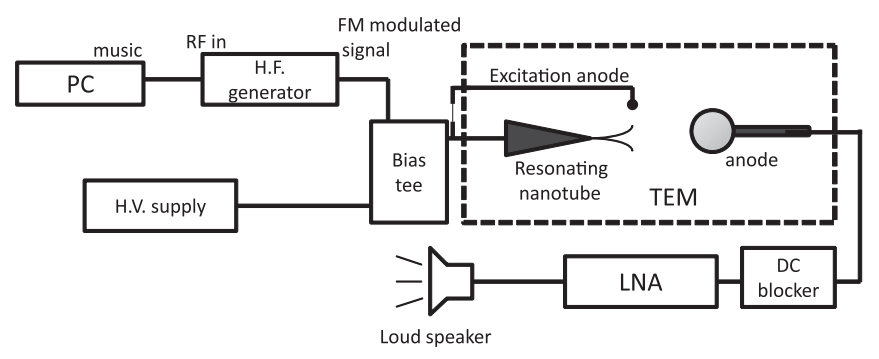

FIG. 5. Schematic showing the configuration used for demodulation.

the resonance no signal was distinguishable although the $d V^{2}$ term should demodulate at any frequency. To distinguish this term from a large noise signal it was necessary to increase the signal amplitude to $V_{\text {ac }} \simeq 2 \mathrm{~V}$. This showed that this term is really negligible in the demodulation performance. Sweeping the carrier's frequency through the resonance gave a large and regular improvement of demodulation up to a maximum at the resonance frequency and a symmetric behavior for the other side of the resonance. As expected, we found a single maximum in the demodulation signal. For $V_{\mathrm{ac}}=0.2 \mathrm{~V}$ and $h=0.9$ we estimate a demodulated current of a few tens of nanoamperes since a $10^{8}$ amplification gain $(10 \mathrm{nA}$ gives $1 \mathrm{~V})$ was used.

In our FE experiments FM demodulation was clearly more difficult than AM in contrast to our recent work on a double-clamped nanoradio based on single-wall nanotube transistors. ${ }^{13}$ For the same CNT it was necessary to increase the excitation voltages to obtain higher amplitudes of vibration in order to hear a distinguishable signal. The minimal values used to obtain an audible signal were $V_{\mathrm{ac}}=0.3 \mathrm{~V}$ and $\omega_{\Delta}=2 \pi \times 10 \mathrm{kHz}$. With such a strong excitation signal the nanotube was driven to a nonlinear duffing regime with hysteresis and jumps. In this regime we found only one maximum of demodulation. The demodulation current was estimated in the range of a few nanoamperes. In the case of a pure note the image shown in Fig. 2(f) was obtained where one observes the two-limit envelopes of vibration that correspond to the amplitudes $y_{1}$ and $y_{2}$ in Fig. 1 .

The signal quality is limited by three main effects. First the instability of the field emission is an important source of noise that is created by atomic diffusion and flip-flop movement on the emitter surface. ${ }^{14}$ Some large changes in the current also appeared in the microampere range due to formation or disappearance of emitting zones (nanoprotrusions) at the apex of the CNT. Normally such strong instabilities would tend to disappear during long-emission experiments but the medium vacuum in the TEM ( $\simeq 10^{-7}$ Torr) compared to the ultrahigh vacuum generally used for FE experiments $\left(\simeq 10^{-10}\right.$ Torr $)$ does not promote this stabilization effect. For AM experiments the higher demodulated currents hide the FE instabilities, but in FM experiments this noise was still significant. The second effect that reduces signal quality is the strong distortion of the signal. This is inherently related to the demodulation process where the mechanical AM variation is multiplied by itself thus leading to harmonic distortion from $2 \omega_{L}$ components. For a complex signal where several frequencies are present we also have intermodulation distortion. This will form additional 

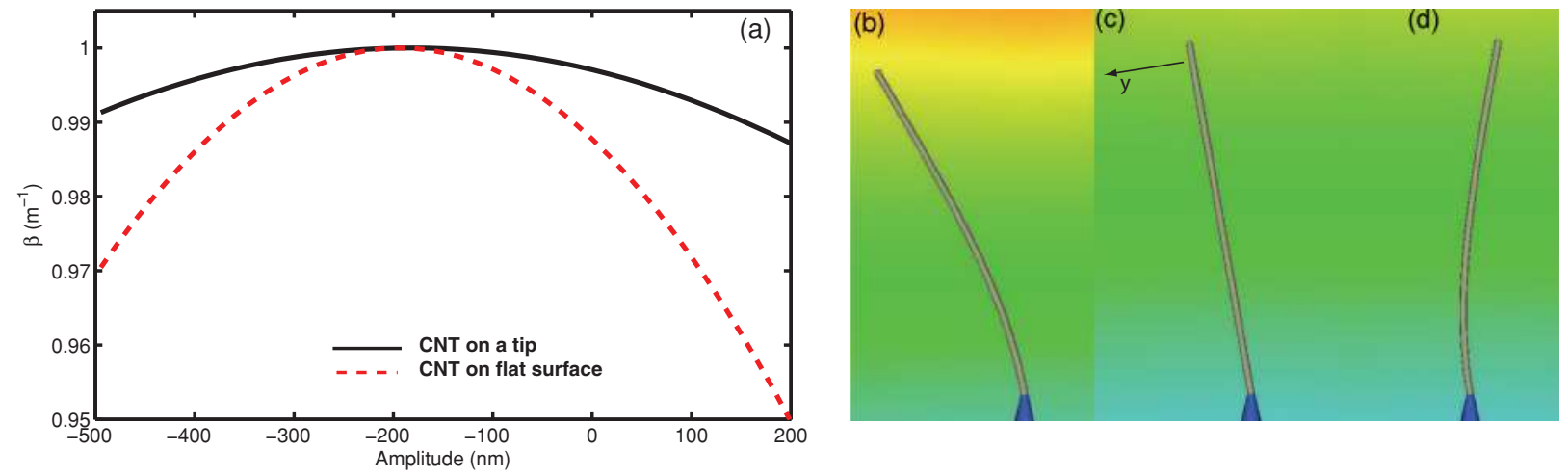

FIG. 6. (Color online) Finite element modelization of the variation of $\beta(y)$ realized using the program Cast $3 \mathrm{M}^{15,16}$ (a) The variation of $\beta$ at the apex (center of the CNT cap) is plotted in red for a CNT at the apex of a support tip and in blue for a CNT on a flat substrate. (b)-(d) Different deformations are simulated using the first eigenmode (with constant length).

signals at frequencies that are not, in general, at harmonic frequencies (integer multiples) of either, but instead often at sum and difference frequencies of the original frequencies. The third effect did a slow decreasing frequency shift of the resonance that altered the experiments, probably due to mass deposition generated by the TEM electron beam.

All the studied nanotubes do not show the same efficiency for demodulation. In particular some CNTs were difficult to excite and higher excitation voltages were necessary to obtain enough amplitude of vibration for distinguishable demodulation. The different reasons for this are discussed below as well as how the efficiency of excitation can be improved to obtain large amplitudes. Another problem for some CNTs is that they emitted very noisy FE currents that lead to strong parasitic effects that totally hide the demodulation current. It is necessary to have cleaner samples without too much amorphous carbon or other instable adsorbates.

\section{SIMULATIONS}

The next step is to determine $\beta(y)$, that is, to obtain $\beta_{1}$ and $\beta_{2}$ in Eq. (4). Systematic numerical simulations were made to determine these parameters taking into account the deformation of the nanotube. $\beta(y)$ can be obtained from the normalized simulations multiplied by the experimental $\beta_{0}$. Simulations have been realized using a free and powerful finite element solver, Cast $3 \mathrm{M},{ }^{15,16}$ adapted to treat electrostatic problems. The model consists of a CNT of length and radius comparable to those of the experiments positioned at the apex of a tip (see Fig. 6). As stated above the nanotube is terminated by a perfect hemisphere despite the fact that more complicated apexes are found experimentally. The simulated nanotube is mounted with a tilt angle of $10^{\circ}$ with respect to the support tip axis. This agrees with the experiments and gives a nonzero value for $\beta_{1}$. From this equilibrium position the nanotube is deformed according to the analytical solution for the first mode of vibration [Figs. 6(b)-6(d)]. For each deformation Cast3m solves the electrostatic problem. $\beta(y)$ for the center of the cap is followed as a function of apex position. Results are presented in Fig. 6(a). The solid black curve represents $\beta(y)$ for the CNT on the tip while the dashed red curve is for the same nanotube on a flat surface. $\beta(y)$ varies quadratically by $\sim 1 \%$ during oscillations for an end angle $\theta \pm 20^{\circ}$. It is asymmetric because of the starting tilt and is maximum for a deflexion of nearly $-200 \mathrm{~nm}$ when the CNT lines up with the support tip axis. For a CNT on a planar surface in the same configuration $\beta(y)$ varies more strongly $(\sim 5 \times)$. This is due to the stronger screening effect of the underneath flat substrate compared to the support tip. This last shows that the specific geometry close to the cantilever is important for optimizing the demodulation.

The values of $\beta_{1}$ and $\beta_{2}$ can be deduced for these geometries by fitting these curves and using the experimental values of $\beta_{0} . \beta_{1}=-9.3539 \times 10^{11} \mathrm{~m}^{-2}$ and $\beta_{2}=-2.5765 \times 10^{18} \mathrm{~m}^{-3}$ were found for the CNT on the tip. Finally, to have a amplitude of vibration of $300 \mathrm{~nm}$ comparable to Fig. 2(c) we estimate $T=4.93 \times 10^{7} \mathrm{~m} \mathrm{~s}^{-2}$ in Eq. (1) (assuming the same $Q=$ 800).

All the necessary parameters for the next part of the simulations are summarized in Table I. With these parameters Eqs. (9) to (12) and Eqs. (13) to (15) can be used to calculate the different demodulation components assuming linear mechanical response.

Figure 7 presents the total demodulated current and the different contributions from an AM rf signal versus the carrier

TABLE I. Parameters used in the simulations on the demodulation performances.

\begin{tabular}{lc}
\hline \hline Parameters & Values \\
\hline$V_{\mathrm{dc}}$ & $300 \mathrm{~V}$ \\
$I_{0}$ & $8 \mu \mathrm{A}$ \\
$B$ & $6.11 \times 10^{10} \mathrm{~V} \mathrm{~m}^{-1}$ \\
$A$ & $1.212 \times 10^{-22} \mathrm{~A} \mathrm{~V}^{-1} \mathrm{~m}^{-1}$ \\
$\beta_{0}$ & $2.905 \times 10^{7} \mathrm{~m}^{-1}$ \\
$\beta_{1}$ & $-9.3539 \times 10^{11} \mathrm{~m}^{-2}$ \\
$\beta_{2}$ & $-2.5765 \times 10^{18} \mathrm{~m}^{-3}$ \\
$Q$ & 800 \\
$\omega_{0}$ & $2 \pi \times 82.0 \mathrm{MHz}$ \\
$\omega_{\Delta}$ & $2 \pi \times 10 \mathrm{kHz}$ \\
$V_{p}$ & $0.3 \mathrm{~V}$ \\
$h$ & 0.9 \\
$T$ & $4.93 \times 10^{7} \mathrm{~m} \mathrm{~s}^{-2}$ \\
\hline \hline
\end{tabular}




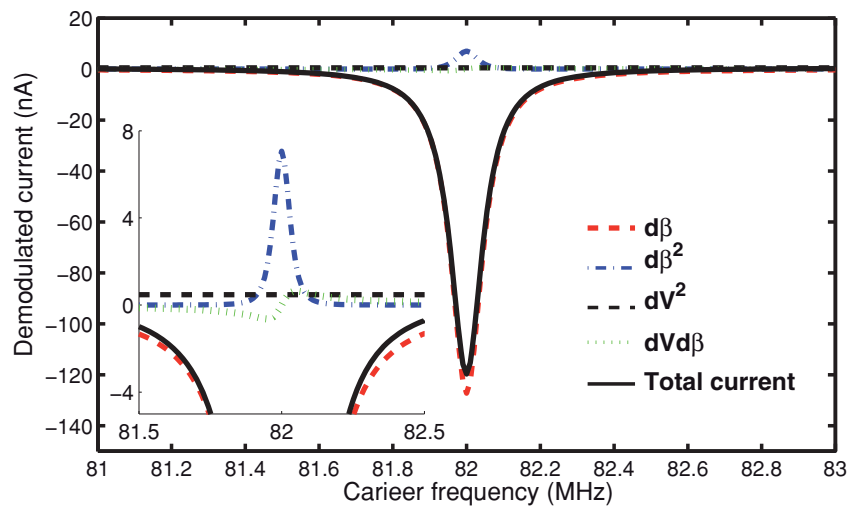

FIG. 7. (Color online) Calculated demodulation currents for an AM signal vs the carrier frequency. Clear maxima at the resonance frequency of the CNT are observed. Most of the demodulation comes from the $d \beta$ term which makes up almost all of the signal. The $d \beta^{2}$ term is the second largest which is of opposite sign, thus reducing slightly the overall demodulation current. The inset is a magnification to better show the smaller and almost negligible contributions.

frequency. As expected we obtain a maximum signal at the resonance frequency reaching $120 \mathrm{nA}$ which is in the range of our experiments. The main demodulating term is clearly the $d \beta$, and thus the $\beta_{2} y^{2}$ dependence of the field amplification factor. All the other terms are negligible except the $d \beta^{2}$ term but as it is of opposite sign compared to the $d \beta$ term, it tends to reduce the overall demodulation performances.

FM demodulation performances are presented in Fig. 8 for the same conditions. Two maxima are observed corresponding to the maximum slope of the response function. The maximum demodulated current is nearly $7 \mathrm{nA}$, more than 10 times lower than AM demodulation. Once again, the $d \beta$ term is the main demodulating term and the $d \beta^{2}$ term decreases somewhat the demodulation. Two antisymmetric peaks were obtained when in linear response but for the high-amplitude nonlinear response, only the first peak is present.

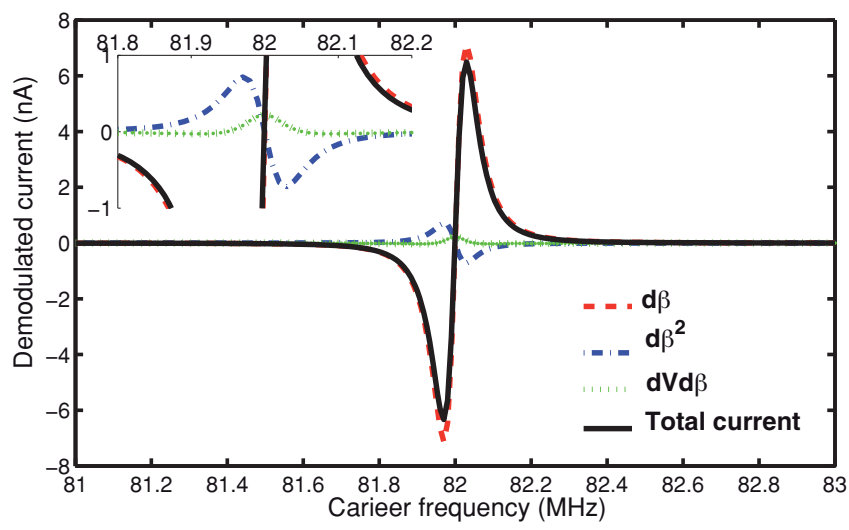

FIG. 8. (Color online) Demodulated current for a FM signal vs the carrier's frequency. We observe two clear maximum at the resonance frequency of the CNT. The main demodulating term is $d \beta$. The second term is $d \beta^{2}$, but since it is of opposite sign it reduces the overall demodulation current. The inset presents a magnification to observe the other contributions that are presently negligible.
Further experiments with a lock-in amplifier to measure the experimental demodulated current versus the carrier frequency were made to compare with these calculations. Unfortunately that run of experiments led only to the destruction of samples or very noisy FE nanotubes so that only low-quality data were obtained. Thus the above analysis still needs an experimental confirmation (demodulated current versus frequency and excitation voltage, etc.) but it already gives clear indications that signal optimization is obtained by maximizing the $d \beta$ term.

\section{DISCUSSION}

Our work clearly shows that the main demodulating term is the variation of $d \beta$ through the $\beta_{2} y^{2}$ dependence of the field amplification factor. The $\beta_{1} y$ dependance used by Jensen et al., that partly contributes in the $d \beta^{2}$, is negligible in this FE configuration. However one can look for conditions under which this term can equal the $\beta_{2}$ term. Comparing Eqs. (8) and (9) or (12) and (13) we see that the two terms are equal if $\beta_{2}=\beta_{1}^{2} / \beta_{0}$. Perhaps a geometry where this condition is satisfied can be found, however since the two contributions are of opposite sign ( $\beta_{2}$ is negative and in the $d \beta^{2}$ term all the $\beta$ parameters are squared) it would tend to lead to an extinction of the demodulation. To improve demodulation one should choose a geometry where one term dominates.

A demodulated current in the $100 \mathrm{nA}$ range was obtained in the simulations for AM modulation which assures a good signal to noise ratio. An estimation of a few nanoamperes was obtained for FM demodulation which is sufficient for transmission but which is more sensitive to noise and instabilities. Experimental work showed roughly the same behavior. However the performances in the two cases vary depending on the choice of the parameters. Comparing Eqs. (9) and (13) we see that the AM signal is proportional to $h y\left(\omega_{c}\right)$ and the FM signal to $\omega_{\Delta} y^{\prime}\left(\omega_{c}\right)$. Consequently increasing $\omega_{\Delta}$ can improve the FM demodulation performance although the simple linear expansion used in our analysis will be wrong. Higher nonlinear terms have the inconvenience of producing additional distortion of the signal.

One way to improve the demodulated current is to increase the dc emission current $I_{0}$ by increasing the dc voltage. However there are two interconnected constraints on this. First the resonance frequency would increase at the same time by the electric field tuning effect which would correspond to changing the radio station. This can in principle be overcome by preselecting the nanotube dimensions or changing its length in situ ${ }^{7,17}$ so that it tunes to the correct frequency at high-emission current. In this work arc electric CNTs with quite high currents of $\sim 10 \mu \mathrm{A}$ were used. However CVD nanotubes that are more compatible with integration and control of their dimensions generally have more defects and thus such high currents can produce self-heating effects ${ }^{18}$ leading to length reduction or even sudden destruction. More generally, the carbon nanotube is the only nanowire form that presently allows such high-field emission currents, stability, and tunability which are necessary for this application. This is intimately related to its material and geometrical properties. 
Now let us consider the nanotube as an antenna that transforms an electromagnetic (em) wave into an electrical circuit signal or vice versa. In their article Jensen et al. proposed that a CNT in the field emission configuration can gain energy from the electromagnetic wave and transform it into a current wave. That is, the electric charge sitting on the CNT due to the FE voltage can be excited by the forces generated by the electromagnetic wave, following $f=q E$, and could lead, if the wave's frequency corresponds to the resonance frequency of the CNT, to large mechanical oscillations. These oscillations cause the $\beta$ variation and lead to a variation in the emission current. Unfortunately our estimation of the forces shows that the nanotube has a very weak response to the original em wave. Consider an em wave with a field $E=0.2 \mathrm{~V} / \mathrm{m}$ that corresponds to the electric field at $1 \mathrm{~km}$ from an isotropic antenna of power $1 \mathrm{~kW}$. The charge on the CNT is obtained from the capacitance $C$ of the CNT. Pushing the simulations one gets optimistically $C=10^{-16} \mathrm{~F}$. For $300 \mathrm{~V}$ that leads to a total charge of $q=3 \times 10^{-14} \mathrm{C}$ and hence to a maximal force of $f=6 \times 10^{-15} \mathrm{~N}$. Assume the most favorable case that all this force is transverse to the CNT axis and located at the apex of the CNT. Using an equivalent spring constant for the first mode of $k=10^{-2}$ $\mathrm{N} / \mathrm{m}$ this leads to a static deflexion of $x_{s}=6 \times 10^{-13} \mathrm{~m}$. At the resonance one gets only $x_{r}=Q x_{s}=6 \times 10^{-10}=0.6 \mathrm{~nm}$ ! Thus, even highly overestimated, the direct excitation of the CNT by a reasonable magnitude em wave is too small to produce a perceptible variation of the field emission current.

An estimation of the capacitive excitation force on the CNT is not simple since it depends strongly on the whole environment of the CNT and on its deformation. The dc voltage bends the CNT from the tilted rest position and this bending in turn modifies the resultant capacitive force. The new position of the CNT corresponds to the equilibrium between electrostatic force and the mechanical restoring force. From this position the rf voltage causes a capacitive ac force that excites the CNT. We performed simulations with Cast3M in the configuration presented in Fig. 6. The dc voltage was $300 \mathrm{~V}$ and the ac voltage was $0.3 \mathrm{~V}$, as can be provided by a external antenna. The excitation forces obtained are in the $10^{-12} \mathrm{~N}$ that is consistent with the observed oscillations with the same applied voltages.

Before applications can be pursued seriously, several points still need to be investigated. First, it would be very interesting to study the use of vertically aligned CNTs grown directly on patterns or flat surfaces, such as can be done by PECVD, because their insertion in sealed components could be easier than arc electric ones. As seen in Fig. 6 this geometry could lead to higher $\beta(y)$ variations. It would be necessary to characterize their performance as demodulators as their physical parameters ( $Q$ factor, tunability, voltage for excitation, amplitude of vibration, etc.) are not known. Such CNTs could also be electrostatically coupled ${ }^{19}$ to increase the demodulated current and increase the $\mathrm{S} / \mathrm{N}$ ratio. Since the coupling between the excitation electrode and the CNT must be as asymmetric as possible for efficiency, an interesting configuration is the one presented in Fig. 9. In this configuration vertical CNT are grown on the sample. The dc applied voltage $V_{A}$ is applied between the CNTs and a upper anode that permits

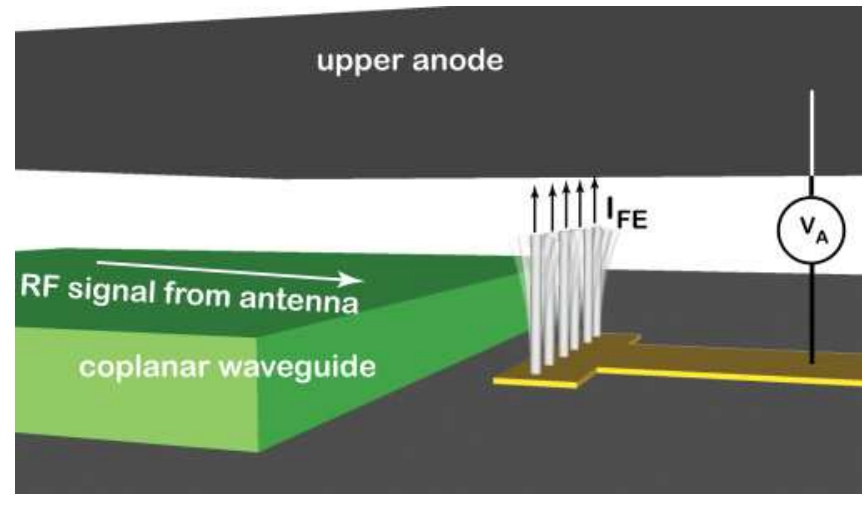

FIG. 9. (Color online) One possible configuration for CNT demodulator component. Vertical CNTs are grown on a substrate and dc voltage $V_{A}$ is applied between CNTs and a upper anode. rf signal received via an external antenna is sent toward the CNTs via a coplanar wideband waveguide. The rf signal component with a carrier frequency matching the mechanical resonance of the CNT excite the nanotubes and the demodulated current is received on the upper electrode.

FE and tuning of the resonance frequency. The rf signal received by an external antenna is sent toward the CNTs via a wideband coplanar waveguide in the vicinity of the CNTs. The low-frequency demodulated signal is then received by the upper anode for further signal treatment.

\section{CONCLUSION}

In conclusion, the equations for AM and FM demodulation by a FE CNT nanoradio have been presented in detail as well as experimental results with measurements or realistic estimations of all the necessary system parameters. Three of the original elements that this in-depth examination have led to are: (1) a corrected and extended mathematical description of the demodulation, (2) a quantitative estimation of all the important governing parameters and their use in calculating the signal generation, and (3) a better understanding of the new antenna concept. More precisely, it has been shown that most of the demodulation effect comes from $\beta(y)$, the dependence of the field amplification factor on the position of the CNT cap. In particular the second-order derivative $\beta_{2}$ is shown to be dominant in contrast to the work of Jensen et $a l .{ }^{7}$ Experimentally and theoretically AM leads to larger modulation currents than FM $(\sim \times 10)$ for the explored range of parameters, though it may be possible to inverse this trend for a different choice of parameters. Estimations of the excitation efficiency of a charged CNT for capturing rf signals have been made and show that this new type of antenna works very poorly in the open FE geometry, that is, for a CNT on a tip. In other words this new type of mechanical reception does not circumvent the basic difficulty of the mismatch between the rf wavelength and CNT length. A better optimization taking into account the electrostatic, electromagnetic, mechanical, and $\mathrm{FE}$ is an engineering problem that must be tackled before the real capabilities and characteristics of this nanoradio are known. 


\section{ACKNOWLEDGMENTS}

This work was supported by French National Research Agency (ANR) through its Nanoscience and Nanotechnology Program (NEXTNEMS, ANR-07-NANO-008-01) and Jeunes
Chercheuses et Jeunes Chercheurs Program (AUTONOME, ANR-07-JCJC- 0145-01). The authors acknowledge the "Plateforme Nanofils et Nanotubes Lyonnaise" of the university Lyon 1. *pascal.vincent@univ-lyon1.fr

${ }^{1}$ S. T. Purcell, P. Vincent, C. Journet, and V. T. Binh, Phys. Rev. Lett. 89, 276103 (2002).

${ }^{2}$ S. Perisanu, A. Ayari, S. T. Purcell, and P. Vincent, Int. J. Nanotechnol. 7, 702 (2010).

${ }^{3}$ C. K. Yanga, A. J. le Fèbre, G. Pandraud, E. van der Drift, and P. J. French, J. Vac. Sci. Technol. B 26, 927 (2008).

${ }^{4}$ K. Jensen, K. Kim, and A. Zettl, Nature Nanotechnol. 3, 533 (2008).

${ }^{5}$ A. Ayari, P. Vincent, S. Perisanu, M. Choueib, V. Gouttenoire, M. Bechelany, D. Cornu, and S. T. Purcell, Nano Lett. 7, 2252 (2007).

${ }^{6}$ H. S. Kim, H. Qin, M. S. Westphall, L. M. Smith, and R. H. Blick, Nanotechnology 18, 065201 (2007).

${ }^{7}$ K. Jensen, H. Garcia, and A. Zettl, Nano Lett. 7, 3508 (2007).

${ }^{8}$ J. M. Kahn, R. H. Katz, and K. S. Pister, J. Commun. Networks 2, 188 (2000).

${ }^{9}$ P. Poncharal, Z. L. Wang, D. Ugarte, and W. A. de Heer, Science 283, 1513 (1999).
${ }^{10}$ M. F. Yu, G. J. Wagner, R. S. Ruoff, and M. J. Dyer, Phys. Rev. B 66, 073406 (2002).

${ }^{11}$ S. Perisanu, P. Vincent, A. Ayari, M. Choueib, S. T. Purcell, M. Bechelany, and D. Cornu, Appl. Phys. Lett. 90, 043113 (2007).

${ }^{12}$ S. Perisanu, V. Gouttenoire, P. Vincent, A. Ayari, M. Choueib, M. Bechelany, D. Cornu, and S. T. Purcell, Phys. Rev. B 77, 165434 (2008).

${ }^{13}$ V. Gouttenoire, T. Barois, S. Perisanu, J. L. Leclercq, S. T. Purcell, P. Vincent, and A. Ayari, Small 6, 1060 (2010).

${ }^{14}$ R. Gomer, Surf. Sci. 38, 373 (1973).

${ }^{15}$ website [http://www-cast 3 m.cea.fr/cast $\left.3 \mathrm{~m}\right]$.

${ }^{16} \mathrm{P}$. Moin, Fundamentals of Engineering. Numerical Analysis (Cambridge University Press, Cambridge, 2001).

${ }^{17}$ P. Poncharal, P. Vincent, J. M. Benoit, S. Perisanu, A. Ayari, M. Choueib, and S. T. Purcell, Nanotechnology 21, 215303 (2010).

${ }^{18} \mathrm{~S}$. T. Purcell, P. Vincent, C. Journet, and V. Thien Binh, Phys. Rev. Lett. 88, 105502 (2002).

${ }^{19}$ S. Perisanu, T. Barois, P. Poncharal, T. Gaillard, A. Ayari, S. T. Purcell, and P. Vincent, Appl. Phys. Lett. 98, 063110 (2011). 